Écrire

l'histoire

\section{Écrire l'histoire}

Histoire, Littérature, Esthétique

18 | 2018

Révolution

\title{
Commémorer Staël et Constant (2017)
}

Enjeux d'un double anniversaire

\section{Léonard Burnand}

\section{OpenEdition}

Journals

Édition électronique

URL : http://journals.openedition.org/elh/1558

DOI : $10.4000 /$ elh.1558

ISSN : 2492-7457

Éditeur

CNRS Éditions

\section{Édition imprimée}

Date de publication : 20 novembre 2018

Pagination : 233-237

ISBN : 978-2-271-12431-9

ISSN : 1967-7499

Référence électronique

Léonard Burnand, «Commémorer Staël et Constant (2017) », Écrire l'histoire [En ligne], 18 | 2018, mis en ligne le 20 novembre 2018, consulté le 16 février 2021. URL : http://journals.openedition.org/elh/ 1558 ; DOl : https://doi.org/10.4000/elh.1558 


\section{Commémorer Staël et Constant (2017)}

Enjeux d'un double anniversaire

Le 18 septembre 1794, alors que la France vient à peine de sortir de la "Grande Terreur», survient la rencontre décisive entre Germaine de Staël et Benjamin Constant, à Lausanne, chez Constance Cazenove d'Arlens, laquelle est à la fois une amie de Germaine et la cousine de Benjamin. Après cette première entrevue, $\mathrm{M}^{\text {me }}$ de Staël écrit à Louis-Adolphe de Ribbing: "J'ai trouvé ici ce soir un homme de beaucoup d'esprit qui s'appelle Benjamin Constant. Pas trop bien de figure, mais singulièrement spirituel. » De son côté, Constant est subjugué par le brio de la fille de Necker, ainsi qu'il le rapportera plus tard dans son récit Cécile: "Je rencontrai, par un hasard qui eut sur ma vie une longue influence, la personne la plus célèbre de notre siècle, par ses écrits et par sa conversation. Je n'avais rien vu de pareil au monde. J'en devins passionnément amoureux. » $\mathrm{Si}$ cette soirée lausannoise constitue le point de départ d'une liaison sentimentale aussi exaltée qu'orageuse, elle est surtout à l'origine d'une complicité intellectuelle extraordinairement féconde, dont l'impact durable sur l'histoire des idées mérite d'être apprécié à sa juste valeur.
Car le couple Staël-Constant a joué un rôle fondamental dans la vie littéraire et politique européenne au tournant des $\mathrm{XVIII}^{\mathrm{e}}$ et XIX ${ }^{\mathrm{e}}$ siècles. Pionniers du romantisme et du libéralisme, opposants à l'autoritarisme napoléonien, défenseurs de la liberté de la presse et de la diversité culturelle, adversaires de l'intolérance religieuse et de la traite des Noirs, précurseurs du roman psychologique et de l'écriture du moi, Germaine et Benjamin ont multiplié les combats en faveur des libertés individuelles. Écrivains engagés, ils ont conçu une œuvre novatrice et foisonnante, qui reste à bien des égards d'une étonnante actualité.

L'année 2017 a permis de célébrer conjointement les deux membres de ce couple d'intellectuels, puisqu'une heureuse coïncidence de calendrier a fait intervenir en même temps le bicentenaire de la mort de Germaine de Staël (Paris, 14 juillet 1817) et les deux cent cinquante ans de la naissance de Benjamin Constant (Lausanne, 25 octobre 1767). Ce double anniversaire a donné lieu à une impressionnante série de publications et de manifestations culturelles, dont il convient de dresser ici un premier bilan. 


\section{Destins croisés : un couple en vitrine}

La double commémoration staëlienne et constantienne a incité les spécialistes des deux auteurs à unir leurs efforts et fédérer leurs ressources, afin d'opérer une synergie entre les deux jubilés et de fêter ceux-ci à l'unisson. Ce projet collectif s'est matérialisé sous la forme d'une exposition et d'un livre-catalogue visant à retracer l'itinéraire de ces deux figures singulières tout en soulignant la variété et l'originalité de leurs écrits. Intitulée Germaine de Stäel et Benjamin Constant, l'esprit de liberté, l'exposition a été présentée du 20 mai au $1^{\text {er }}$ octobre 2017 dans le cadre de la Fondation Martin Bodmer (Genève). Le commissariat scientifique a été confié aux deux initiateurs du projet : le soussigné, directeur de l'Institut Benjamin Constant (université de Lausanne) et Stéphanie Genand, présidente de la Société des études staëliennes (Paris). Le livrecatalogue qui accompagnait cette exposition a paru sous le même titre aux éditions Perrin, en un volume réunissant une vingtaine d'essais rédigés par des chercheurs issus de différents horizons géographiques et disciplinaires, ainsi qu'une iconographie abondante et souvent inédite. Mettant à profit plusieurs types de supports (manuscrits, livres anciens, tableaux, gravures, objets...), ce parcours instaure un dialogue dynamique entre textes et images, de manière à éclairer sous un nouveau jour, à travers deux destins croisés, une époque durant laquelle s'est dessiné l'avenir de l'Europe, entre Lumières, Révolution, Empire et Restauration. Envisagée de la sorte, la trajectoire du couple StaëlConstant sert de fil conducteur pour revisiter une période charnière de notre histoire et en incarner certains traits significatifs. Divisés en quatre volets (l'enfance et la formation intellectuelle ; l'expérience de la Révolution ; l'action et la pensée politiques ; la création littéraire entre intime et fiction), l'exposition et l'album s'emploient à mettre en évidence les fortes convergences idéologiques et esthétiques entre l'auteure de Corinne et l'auteur d'Adolphe, tout en examinant les particularités et les apports spécifiques de chacun. Si Germaine de Staël avait déjà fait l'objet d'une exposition importante en 1966, de même que Benjamin Constant en 1967 et 1980, ces deux personnages n'avaient encore jamais été abordés ensemble dans une même exposition. Le fait de placer leur couple au centre du dispositif a permis de renouveler les approches thématiques et les sources mobilisées. Le succès de l'exposition de 2017 auprès du public et l'ampleur de la couverture médiatique de cet événement culturel montrent qu'une telle façon d'écrire l'histoire permet de capter l'attention d'un large auditoire : la dimension sentimentale et romanesque dont l'aventure d'un « couple en Révolution » est porteuse a sans doute servi d'appât pour attirer à la Fondation Martin Bodmer des milliers de visiteurs qui, une fois sur place, ont aussi pu être initiés à des aspects plus conceptuels de l'histoire politique et intellectuelle du tournant des Lumières. Le cœur conduit parfois à la raison... 


\section{Germaine et Benjamin en librairie}

L'un des effets les plus visibles de ce double anniversaire fut la forte présence de Staël et Constant sur les rayons des librairies. Il faut dire que l'année 2017 a vu se succéder à une cadence élevée bon nombre de publications staëliennes et constantiennes. Outre le livre-catalogue consacré au couple et mentionné ci-dessus, plusieurs essais ont paru, à commencer par La Chambre noire (Genève, Droz), un ouvrage dans lequel Stéphanie Genand explore de façon originale l'ensemble du corpus de Germaine de Staël, en démontrant que cette œuvre si souvent associée à la notion d'enthousiasme se construit en même temps sur un "manque", une faille, et de profonds silences qui contredisent l'idée reçue selon laquelle la « trop célèbre » aurait été perpétuellement avide d'éclat, de bruit et de reconnaissance. Dans un autre registre, le recueil de Jean Goldzink et Gérard Gengembre, Madame de Staël, la femme qui osait penser (Classiques Garnier), et le volume de Michel Aubouin, Madame de Staël ou l'Intelligence politique (Omnibus), mettent en exergue la modernité et l'esprit européen de la baronne de Coppet. Un phénomène éditorial marquant de cette séquence commémorative s'est manifesté dans le puissant effort de mise à disposition des textes de Staël et Constant auprès d'un public élargi. En avril, l'entrée remarquée de $\mathrm{M}^{\mathrm{me}}$ de Staël dans la Pléiade a constitué l'une des étapes saillantes du jubilé. Tandis que Benjamin avait intégré la prestigieuse collection de Gallimard dès 1957, Germaine n'avait pas encore eu droit à son volume. Catriona Seth, en collaboration avec Valérie Cossy, a comblé cette importante lacune en réunissant sur papier bible les deux romans staëliens (Delphine et Corinne) et le traité De la littérature. Au même moment, Laurent Theis et Michel Winock ont rassemblé les principaux écrits politiques de $\mathrm{M}^{\mathrm{me}}$ de Staël dans une anthologie de plus de mille pages intitulée La Passion de la liberté (R. Laffont, coll. "Bouquins»). En mai, alors qu'on inaugurait l'exposition Staël-Constant à la Fondation Martin Bodmer, Stéphanie Genand et Jean-Daniel Candaux ont fait paraître chez Champion-Slatkine les deux derniers volumes de la Correspondance générale de Staël, qui couvrent les années 1812-1817; ces tomes VIII et IX contiennent de nombreuses lettres inédites qui jettent un éclairage inattendu sur la période tardive de l'existence de la femme-auteur. Toujours en mai, la collection «Folio. Classique» a accueilli une nouvelle édition du roman épistolaire Delphine, sous la conduite d'Aurélie Foglia. Entre le printemps et l'automne, la série des CEuvres complètes de Madame de Stä̈l (Champion) s'est enrichie de deux imposants volumes : l'édition des Considérations sur les principaux événements de la Révolution française établie par Lucia Omacini et Stefania Tesser sur la base du manuscrit original conservé à la Bibliothèque nationale de France, et l'édition du grand traité De l'Allemagne présentée et annotée par Axel Blaeschke. Du côté constantien, l'année 2017 a également généré une belle moisson éditoriale. On relèvera en particulier l'édition commentée des Journaux intimes de Benjamin Constant réalisée par JeanMarie Roulin (Gallimard, coll. "Folio. Classique »), qui rend ces écrits personnels accessibles à un lectorat plus large 
et démontre que l'auteur d'Adolphe et des Principes de politique ne fut pas seulement un maître du roman d'analyse et un des fondateurs du libéralisme, mais aussi un diariste de tout premier plan. Dans la série des CEuvres complètes de Benjamin Constant (De Gruyter), Kurt Kloocke et Paul Delbouille ont dirigé le tome $\mathrm{XV}$, qui réunit les brochures politiques publiées par Constant au début de la seconde Restauration, ainsi que son fameux texte sur la liberté des Anciens et des Modernes. Le contexte du deuxcent-cinquantenaire de la naissance de Benjamin a également incité le Liberty Fund (États-Unis) à publier une traduction en anglais du Commentaire sur l'ouvrage de Filangieri, une des pièces maîtresses de la théorie politique de Constant, tandis que l'historien du droit Marco Caldeira a fait paraître à Lisbonne (chez l'éditeur Chiado) un essai qui met en lumière l'influence des idées constantiennes sur le constitutionnalisme portugais. Enfin, les deux revues spécialisées dans l'étude de Staël et Constant (les Cahiers staëliens et les
Annales Benjamin Constant) ont chacune élaboré un numéro spécial à l'occasion du double anniversaire.

Ce cortège de publications s'est accompagné d'une succession de colloques et journées d'étude sur Germaine de Staël et/ou Benjamin Constant, qui se sont tenus tout au long de l'année dans différents pays : de l'Institut français de Berlin à l'université de Naples en passant par la Fondation Napoléon, l'École normale supérieure de Lyon, I'UNESCO, le Château de Coppet, l'Opéra de Lausanne, l'université de Bucarest, la Chawton House Library ou l'université de Virginie, de nombreuses institutions culturelles ont consacré des manifestations scientifiques et des cycles de conférences aux deux écrivains libéraux, dont la notoriété dépasse de plus en plus les frontières de la francophonie, ce qui témoigne d'une internationalisation croissante des études sur Staël et Constant (plusieurs textes politiques et littéraires de ce dernier ont récemment fait l'objet de traductions en japonais ou en persan).
Alors que notre époque se caractérise par une inflation des commémorations et qu'on assiste à un défilé ininterrompu de célébrations historiques en tous genres, au point que certains évoquent une «frénésie commémorative " (Étienne François) ou une « commémorationnite » (Pierre Assouline), on peut légitimement s'interroger sur la nécessité de mettre en œuvre un programme d'une telle ampleur pour fêter le couple StaëlConstant. L'auteur de ces lignes n'est sans doute pas le mieux placé pour se prononcer sur cette question, étant donné qu'il a lui-même été l'un des principaux artisans de ce double anniversaire! Il est vrai qu'au cœur du déluge mémoriel qui nous entoure, l'omniprésence des commémorations peut parfois s'avérer lassante, voire assommante. Toutefois, il existe aussi un «bon usage des commémorations " (Bernard Cottret et Lauric Henneton). Lorsque la célébration d'un anniversaire ne constitue pas une fin en soi, mais qu'elle est au service d'une vaste remise à jour des connaissances 
(notamment à travers des éditions critiques de textes et des rencontres scientifiques interdisciplinaires) et d'un véritable effort de médiation culturelle et de vulgarisation de qualité, une telle pratique commémorative peut se révéler utile et fructueuse. Le double anniversaire de Staël et Constant vient d'en apporter - du moins l'espérons-nous une stimulante démonstration. Il faut dire que les festivités staëliennes et constantiennes de 2017 sont intervenues dans une conjoncture particulièrement favorable, étant donné que l'esprit de Coppet véhiculé par l'œuvre de Germaine et Benjamin correspond assurément à un certain air du temps : dans leur recherche d'une synthèse progressiste entre l'ordre et la liberté, dans leur refus des extrêmes et leur volonté de trouver une voie médiane et modérée capable d'unifier le centre gauche et le centre droit, dans leur tentative de tracer les contours d'une Europe plurielle et revivifiée, Staël et Constant ont en effet forgé une pensée susceptible de rencontrer aujourd'hui une puissante résonance dans notre espace public et médiatique. Voilà qui nous rappelle opportunément que le phénomène commémoratif est intimement lié au contexte dans lequel il se déploie. Pour reprendre l'heureuse formule de Pierre Nora, «l'histoire propose, mais le présent dispose ». 